\title{
Parásitos gastrointestinales de aves silvestres en un ecosistema ribereño urbano tropical en Heredia, Costa Rica
}

\author{
Gabriela Pérez-Gómez ${ }^{1}$, Ana Eugenia Jiménez-Rocha ${ }^{1} \&$ Tania Bermúdez-Rojas ${ }^{1}$ \\ 1. Universidad Nacional, Heredia, Costa Rica. Apdo 86-3000. gabytta1985@gmail.com; ana.jimenez.rocha@una.cr; \\ tania.bermudez.rojas@una.cr
}

Recibido 23-X-2017. Corregido 12-III-2018. Aceptado 12-IV-2018.

\begin{abstract}
Gastrointestinal parasites of wild birds in a tropical riverine urban ecosystem in Heredia, Costa Rica. In urban ecosystems, rivers provide various ecosystem services, among them, serving as interurban biological corridor, allowing birds to move and shelter in the middle of the city. However, high levels of pollution expose them, and even humans, to several health problems. Hence the importance of performing environmental diagnoses that allows to identify alterations and mitigate them in a timely manner. This study provides a first diagnosis of the parasitic situation of wild birds along the Pirro river which is located in an urban environment, fragmented and with high levels of surface water pollution. The sampling was carried out at the upper and middle part of the Pirro river in Heredia, Costa Rica. Mist-nets were used to capture the birds, and a general objective exam (GOE) was conducted in order to evaluate their nutritional and hydration state, as well as their physical state. In addition, stool samples were collected in order to characterize the gastrointestinal parasites (GPI). This procedure was carried out on 158 birds, belonging to 30 species, three PGI groups were identified: protozoa, nematodes and cestodes. The highest percentages of infection were represented by coccidia, with 97.6 $\%$, followed by nematodes and cestodes with $2.4 \%$. Within the group of coccidios, it was possible to identify Eimeria spp., Isospora spp., in the nematodes: at the Strongylida group level and at the Capillaria spp., and for the cestodes: Choanotaenia spp. GOE resulted regular-to-good in all individuals captured, however, a general linear model was used to identify that the oral cavity and the state of the plumage could be the more linked variables with the percentage of infection by gastrointestinal parasites in birds, due to the fact that the oral cavity is the bird's main route of ingestion, as well as the state of the plumage shows its vigor. Rev. Biol. Trop. 66(2): 788-798. Epub 2018 June 01.
\end{abstract}

Key words: urban birds; gastrointestinal parasites; urban rivers; wild birds.

En los ecosistemas urbanos, los ríos son uno de los elementos articuladores en el desarrollo de las ciudades cumpliendo una función ecológica muy importante, actuando como corredores biológicos interurbanos donde la vida silvestre se traslada y refugia. Actualmente, estos ecosistemas son poco preservados, ya que el ser humano se establece más exitosamente que otras especies, aumentando la fragmentación del paisaje natural (Barrientos \& Monge-Nájera, 2010; Monge-Nájera, 2013).

La contaminación y el inadecuado manejo de los residuos sólidos que a diario se produce en las zonas urbanas, es una posible consecuencia de la proliferación de agentes infecciosos, tanto en seres humanos como en la fauna silvestre (Azpiri, Maldonado, \& González 2000). Pese a la contaminación existente, las aves son uno de los grupos taxonómicos que logra obtener beneficios en las zonas de protección de los ríos urbanos, ya que es donde realizan la mayor parte de sus actividades, tales como: forrajeo, reproducción, zonas de paso, entre otras. Sin embargo, los altos niveles de contaminación de algunos ríos urbanos las exponen a adquirir enfermedades que podrían ocasionarles la 
muerte (Friend \& Franson, 1999; Azpiri et al. 2000; Freitas, Oliveira, Cavalcanti, Leite, Magalhaes, Oliveira, \& Evencio-Sobrinho, 2002; Tomás-Cabedo, Merino, Moreno, Morales, \& Martínez-De La Puente, 2007; Papazahariadau, Diakou, Papadopoulos, Georgopoulou, Komnenou, \& Antoniadou-Sotiriadou, 2008).

Muchas enfermedades comunes en aves son ocasionadas por parásitos, viéndose favorecidas por fuentes de agua contaminada, como los ríos. Estos representan medios de transmisión de parásitos entre las aves y el ser humano o viceversa, a través de la zoonosis (Friend \& Franson, 1999; Freitas et al. 2002; Papazahariadau et al. 2008).

Entre los endoparásitos más comunes de las aves se encuentran los nematodos (gusanos redondos), cestodos (tenias), trematodos (duelas), acantocéfalos (gusanos tornillo) y protozoos (coccidios, entre otros) (Loot, Park, Lek, $\&$ Brosse, 2006). La mayoría de los nematodos y protozoarios tienen un ciclo de vida directo, mientras que los cestodos tienen ciclo de vida indirecto con la participación de huéspedes intermediarios (Freitas et al. 2002).

En Suramérica se han realizado estudios parasitológicos en aves de cautiverio, como zoológicos o aviarios, permitiendo identificar patógenos específicos de familias de aves (Freitas et al. 2002; Hernández, Larramend, \& Szczypel, 2002; Hinojosa-Sáenz, \& González-Acuña, 2005; Marín-Gómez \& Benavides-Montaño, 2007; Rojas, Larramendy, Sánchez, González-Acuña, Lara, \& Cicchino, 2009; Mena \& Neyra, 2009; García-Corredor, Sánchez-Parada, Pulido-Medellín, \& AndradeBecerra, 2013). En Colombia, fue reportado en aves de la familia Psittacidae, los parásitos Capillaria spp. y Ascaridia spp.; así como protozoarios Isospora spp., los cuales produjeron pérdida de peso, debilidad, anorexia, plumaje erizado y problemas en sus ciclos reproductivos (Burbano, Acosta, Montaño, \& Martínez, 2003).

En Costa Rica, la mayoría de los estudios sobre parásitos gastrointestinales (PGI) han sido realizados en aves silvestres de cautiverio (Campos, 1997; List \& Solano, 2001; Arce,
2005; Sibaja, 2006; Jiménez 2016, com. pers.); pero existen escasos reportes de PGI en aves silvestres de zonas urbanas. En palomas y en aves paseriformes se ha reportado Ascaridia columbae, Raillietina echidnobothrida e Eimeria spp. (Jiménez, Wearing, Hernández, Di Mare, Villareal, Cedeño, \& Ramírez, 2002; Rodríguez-Ortíz, García-Prieto, \& Pérez-Ponce de León, 2004), mientras que en aves de plantaciones de café se han reportado acantocéfalos, Syngamus trachea e Isospora zorzai (Keller, Yabsley, Gibbs, McGraw, \& Hernández 2012; Hernández, Peters, Weygandt, Jiménez, Villegas, O'Connor, Michael, Yabsley, García, \& Carroll, 2013); y en zanates de parques urbanos se reportaron acantocéfalos, cestodos sin identificar e Isospora spp. (Picado \& Castillo 2007). En un estudio reciente en aves paseriformes de la zona Sur del país se reportó Acuaria spp., Capillaria spp., ascaridios y coccidios (González, 2016).

Sin embargo, hasta la fecha ningún estudio parasitológico en aves ha sido realizado en ríos urbanos de Costa Rica, por lo que surge la necesidad de realizar un primer diagnóstico de los PGI presentes en las aves silvestres que habitan el ecosistema ribereño de la microcuenca urbana del río Pirro (Heredia). Esta microcuenca presenta serios problemas ambientales, tales como contaminación de las aguas superficiales, impermeabilización de los suelos, desbordamiento del cauce y pérdida de la cobertura boscosa, lo cual reduce la disponibilidad de recursos para las aves (Romero, Piedra, Villalobos, Marín, \& Núñez 2011; Alvarado, Bermúdez, Romero, \& Piedra, 2014).

\section{MATERIALES Y MÉTODOS}

Área de estudio: El río Pirro se ubica en la provincia de Heredia, Costa Rica y es un tributario intermitente del río Bermúdez, afluente de la subcuenca del río Virilla, dentro de la cuenca del río Grande de Tárcoles. Tiene una extensión de $7.36 \mathrm{~km}^{2}$ con una longitud total de $9.7 \mathrm{~km}$, se encuentra a 1050 y $1420 \mathrm{msnm}$, con temperaturas que oscilan entre los 15 y $25^{\circ} \mathrm{C}$. La microcuenca del río Pirro está conformada 
por un $73 \%$ de área urbana, $5.6 \%$ vegetación ribereña y $16.3 \%$ de cultivos de café, con tendencia a crecer en el área urbana (Romero et al. 2011; Alvarado et al. 2014).

Se seleccionaron cuatro sitios de estudio desde la parte alta del río Pirro hasta la parte media (Fig. 1), ubicados en dos cantones (San Rafael y Heredia) y tres distritos (Los Ángeles, Santiago y Heredia).

En la parte media de la microcuenca del río Pirro se seleccionaron tres sitios de estudio, el primero se ubicó en Guayabal, específicamente en los jardines de la Iglesia Centro Cristiano de Heredia $\left(09^{\circ} 59^{\prime} 34.3\right.$ ” N, 8406'49.1” W), a una altitud de $1140 \mathrm{msnm}$. El segundo en la Universidad Nacional, dentro del jardín universitario del antiguo Centro de Documentación en Vida Silvestre $\left(09^{\circ} 59^{\prime} 54.9^{\prime \prime}\right.$ N, 8406'34.1" W) a una elevación de $1174 \mathrm{msnm}$. El tercer sitio en el cantón de Heredia, distrito Santiago, en una plantación privada de café $\left(10^{\circ} 00^{\prime} 21.3^{\prime \prime}\right.$ $\left.\mathrm{N}, 8^{\circ} 06^{\prime} 16.3^{\prime \prime} \mathrm{W}\right)$ a una elevación de 1171 msnm. Finalmente, en la parte alta de la microcuenca, se ubicó el cuarto sitio, también en una plantación privada de café $\left(10^{\circ} 01^{\prime} 56.6^{\prime \prime} \mathrm{N}\right.$, $\left.84^{\circ} 05^{\prime} 32.8^{\prime \prime} \mathrm{W}\right)$ a una altura de $1450 \mathrm{msnm}$.
Captura de aves: Se realizó durante la época seca (febrero-abril) y lluviosa (agostosetiembre) del 2011. Se utilizaron seis redes de niebla en cada sitio, con dimensiones estándar, y un diseño experimental basado en la metodología para uso de redes descrita por Ralph et al. (1996), modificándola para zonas urbanas en cuanto al tiempo de revisión de redes (de 10 a 15 minutos) y horas de apertura durante la mañana: 6:30-11:30 y por la tarde: 13:3016:30. Una vez capturadas las aves se colocaron en bolsas de tela hasta su posterior evaluación.

Parámetros de salud en las aves: Una vez capturada el ave se procedió a hacer una descripción de algunos parámetros de salud como el: Examen Objetivo General (EOG) y la presencia de PGI.

El EOG consistió en revisar la totalidad del cuerpo del ave, mediante el tacto o palpación de los órganos y extremidades superiores (alas) e inferiores (patas) (Brejov, 2014). Se utilizó una escala de evaluación entre uno a tres, donde uno fue bueno, dos fue regular y tres fue malo. Las partes evaluadas del cuerpo del ave fueron: ojos y cavidad oral (hidratado o deshidratado de acuerdo al estado de las mucosas), quilla

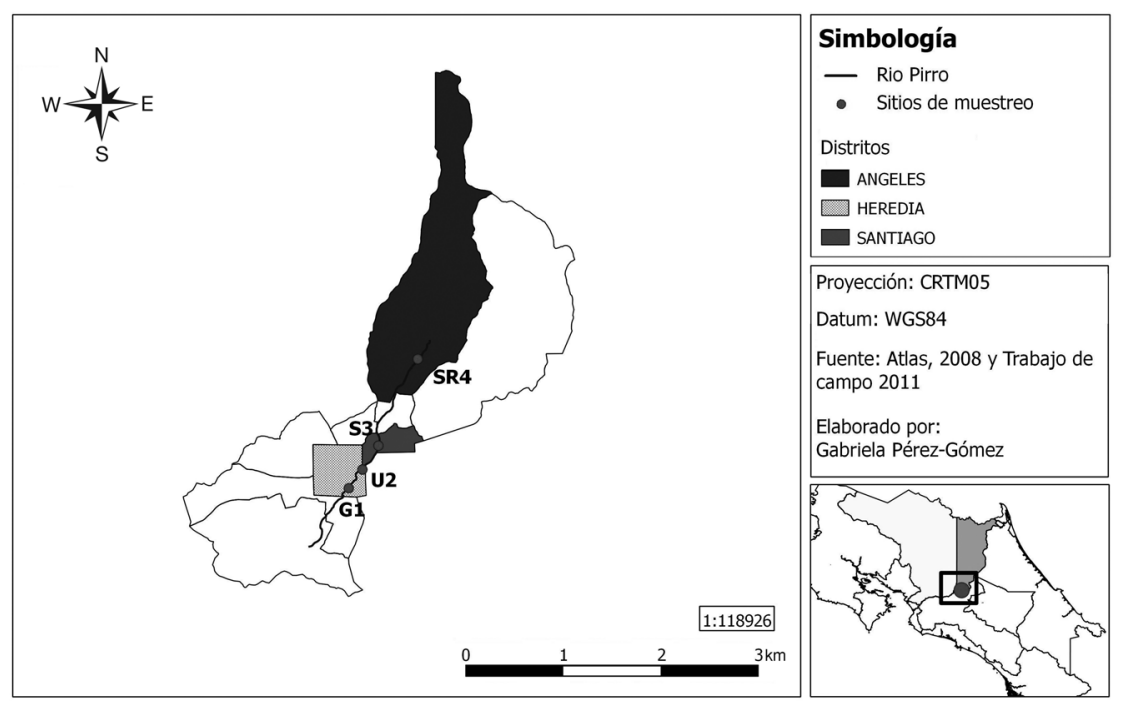

Fig. 1. Ubicación de los sitios de estudio: Guayabal (G1), Universidad Nacional (U2), Santiago (S3) y San Rafael (SR4) del río Pirro, Heredia, Costa Rica. 
(mediante la palpación se logró aproximar indicios de desnutrición), región abdominal (mediante palpación se percibieron protuberancias anormales), patas (presencia/ausencia de deformaciones o falta de miembros), cloaca (presencia/ausencia de restos de heces para identificar indicios de cuadros diarreicos) y alas (problemas mecánicos). También se realizaron algunas mediciones biométricas como: desgaste del plumaje (se utilizó una escala de cero a tres (donde cero representa ningún desgaste, uno desgaste leve, dos desgaste moderado y tres desgaste grave), edad y sexo (cuando era posible identificarlos), medida del ala y peso.

La presencia de PGI fue evaluada a partir de la recolecta de heces producto de la defecación espontánea cuando estuvieron dentro de la bolsa de tela, la cual fue lavada en cada muestreo para evitar la contaminación. Las heces se recolectaron directamente con un hisopo de madera, se colocaron en viales eppendorf con $0.5 \mathrm{ml}$ de solución salina, conservadas a $4{ }^{\circ} \mathrm{C}$ en una hielera. Posteriormente se trasladaron al Laboratorio de Parasitología de la Escuela de Medicina Veterinaria de la Universidad Nacional para su posterior análisis. Las muestras fueron procesadas por medio de dos técnicas: directo salina-lugol, para detectar protozoarios como Giardia duodenalis; y la técnica de Sheather con solución hipersaturada de azúcar para detectar ooquistes de coccidios y huevos de nematodos. La identificación se realizó a nivel de grupo o género de acuerdo a lo descrito por Sloss, Kemp y Zajac (1994) y Foreyt (2001).

Todas las aves capturadas se anillaron, y en el caso de las recapturas en muestreos diferentes, se evaluaron nuevamente los parámetros de salud.

El porcentaje de infección por PGI se calculó con la siguiente fórmula: $\boldsymbol{P I}=\boldsymbol{N}^{+} /$ $N^{T} * 100$, donde $P I=$ porcentaje de infección, $\boldsymbol{N}^{+}=$número de pruebas positivas de PGI, $\boldsymbol{N}^{\boldsymbol{T}}=$ número de individuos por especie.

La relación entre el EOG de las aves y el porcentaje de infección por PGI, se realizó mediante el modelo de regresión lineal general (General Lineal Model), con la ecuación glm(Porcentaje_infección $\sim$ Cavidad_oral + Quilla + Patas + Alas + Plumaje, family=gaussian(identity), data=datos) $\mathrm{y}$ una función gausiana. Para la elección del mejor modelo se utilizó el Criterio de Información de Akaike (Information Criterion), permitiendo utilizar la máxima verosimilitud y el mejor ajuste de los datos (Posada y Rosero 2007), mediante el programa estadístico $R$ Comander (2008).

Parámetros físicos y bacteriológicos del agua: Se realizó un estudio descriptivo en cuanto a la calidad del agua superficial del río, tomando variables fisicoquímicas del agua como: Oxígeno disuelto $(\mathrm{mg} / \mathrm{l})$, porcentaje de saturación de oxígeno $(\%)$, temperatura $\left(\mathrm{C}^{\circ}\right)$ y pH, realizándolo una vez en dos épocas del año (lluviosa y seca), con un multiparámetro Thermo Scientific Orion 5 Star.

Para estimar la carga bacteriológica, se tomó una única muestra de agua en época seca y otra en lluviosa, con recolectores estériles de $100 \mathrm{ml}$ rotulados previamente. Las muestras se mantuvieron en una hielera a $10{ }^{\circ} \mathrm{C}$ aproximadamente y se trasladaron al laboratorio de bacteriología de la Escuela de Medicina Veterinaria de la Universidad Nacional para su procesamiento. A estas muestras se les realizó el análisis microbiológico de coliformes fecales y Escherichia coli siguiendo el protocolo de laboratorio de Clesceri, Greenberg y Trussell (1989).

Para el análisis de los datos generados por el laboratorio de bacteriología, se utilizó un índice de Calidad de Agua (Oram 2013), dado por la Fundación Sanitaria Nacional (NSF, National Sanitation Foundation), en Dallas, Estados Unidos de Norteamérica, el cual clasifica la calidad, así: 90-100 excelente, 70-90 buena, 50-70 media, 25-50 mala y 0-25 muy mala.

Las mediciones de la calidad del agua realizadas se reportan de forma descriptiva para visualizar el nivel de contaminación de los sitios de estudio, y además son puntuales, lo que significa que dependieron de las condiciones del agua en el momento de la toma. 


\section{RESULTADOS}

Parámetros de salud de aves: Se evaluaron 158 individuos correspondientes a 30 especies de aves capturadas en el río Pirro (Cuadro 1). El $52.5 \%$ (83 individuos) de las aves capturadas mostró un EOG bueno, mientras que un $42.4 \%$ (67 individuos) y un $5.0 \%$ (8 individuos) fue regular y malo respetivamente. Los principales signos clínicos observados en las tres categorías del EOG fueron: bajo peso, deshidratación y diarrea; sin embargo, en una de las especies de aves (Turdus grayi) se observaron malformaciones, específicamente en la región del pico y patas, así como mutilaciones o protuberancias en los corvejones y falanges.

El $25.9 \%$ (41/158) de las aves capturadas fueron positivas a PGI, representando un $50 \%$ $(15 / 30)$ de las especies analizadas. El total de las muestras positivas a PGI correspondieron a parásitos no zoonóticos. Se identificaron tres grupos de estos: protozoarios, nematodos y cestodos. Los porcentajes de infección más altos estuvieron representados por los coccidios, con $97.6 \%$ (40/41) (sin esporular/esporulados). Dentro del grupo de los coccidios un

CUADRO 1

Especies de aves capturadas en el río Pirro, Heredia, 2011

\begin{tabular}{|c|c|c|c|}
\hline Familia & Especie & Nombre en inglés & Nombre en español \\
\hline Columbidae & Columbina inca & Inca Dove & Tortolita Colilarga \\
\hline Columbidae & Leptotila verreauxi & White-tipped Dove & Paloma Coliblanca \\
\hline Columbidae & Zenaida asiatica & White-winged Dove & Paloma Aliblanca \\
\hline Momotidae & Momotus lessonii & Lesson's Motmot & Pájaro Bobo, Bobo Azul \\
\hline Picidae & Melanerpes hoffmannii & Hoffmann's Woodpecker & Carpintero de Hoffmann \\
\hline Tyrannidae & Contopus sp & - & Pibí \\
\hline Tyrannidae & Megarynchus pitangua & Boat-billed Flycatcher & Mosquerón Picudo \\
\hline Tyrannidae & Pitangus sulphuratus & Great Kiskadee & Bienteveo Grande \\
\hline Vireonidae & Vireo flavoviridis & Yellow-green Vireo & Vireo Cabecigrís \\
\hline Vireonidae & Vireo philadelphicus & Philadelphia Vireo & Vireo Amarillento \\
\hline Vireonidae & Vireo olivaceus & Red-eyed Vireo & Vireo Ojirrojo \\
\hline Troglodytidae & Troglodytes aedon & House Wren & Soterrey Cucarachero \\
\hline Troglodytidae & Cantorchilus modestus & Cabanis's Wren & Soterrey Chinchirigüí \\
\hline Turdidae & Turdus grayi & Clay-colored Thrush & Mirlo Pardo (Yigüirro) \\
\hline Turdidae & Catharus aurantiirostris & Orange-billed Nightingale-Thrush & Zorzal Piquianaranjado \\
\hline Turdidae & Catharus ustulatus & Swainson's Thrush & Zorzal de Swainson \\
\hline Passerellidae & Melozone cabanisi & Cabanis's Ground-Sparrow & Pinzón Cafetalero \\
\hline Passerellidae & Melozone leucotis & White-eared Ground-Sparrow & Pinzón Orejiblanco \\
\hline Passerellidae & Zonotrichia capensis & Rufous-collared Sparrow & Chingolo (Comemaíz) \\
\hline Passerellidae & Atlapetes albinucha & White-naped Brushfinch & Saltón Gargantiamarilla \\
\hline Parulidae & Basileuterus rufifrons & Rufous-capped Warbler & Reinita Cabecicastaña \\
\hline Parulidae & Setophaga petechia & Yellow Warbler & Reinita Amarilla \\
\hline Parulidae & Parkesia noveboracensis & Northern Waterthrush & Reinita Acuática Norteña \\
\hline Parulidae & Cardellina canadensis & Canada Warbler & Reinita Pechirrayada \\
\hline Parulidae & Cardellina pusilla & Wilson's Warbler & Reinita Gorrinegra \\
\hline Parulidae & Protonotaria citrea & Prothonotary Warbler & Reinita Cabecidorada \\
\hline Parulidae & Oreothlypis peregrina & Tennessee Warbler & Reinita Verdilla \\
\hline Cardinalidae & Piranga rubra & Summer Tanager & Tangara Veranera \\
\hline Thraupidae & Thraupis episcopus & Blue-gray Tanager & Tangara Azuleja \\
\hline Thraupidae & Saltator coerulescens & Grayish Saltator & Saltator Grisáceo \\
\hline
\end{tabular}


$14.6 \%(6 / 41)$ y un $2.4 \%(1 / 41)$ fue positivo a Isospora spp. e Eimeria spp., respectivamente.

Strongylida, Capillaria spp, y Choanotaenia spp., para el grupo de los nematodos y cestodos respectivamente, cada uno presentó un porcentaje de infección $2.4 \%$ de (Apéndice digital 3). Las infecciones simples (98.7\%) fueron las más frecuentes, representadas por los coccidios (27 individuos) o por Isospora spp. (4 individuos); mientras que la infección mixta se presentó en una única especie de ave (T. grayi), donde se diagnosticaron los 4 tipos de PGI encontrados en este estudio (Cuadro 2).

Finalmente, según el modelo de regresión lineal (General Lineal Model), se logró demostrar que la cavidad oral $(\mathrm{P}=0.00115)$ y las alas $(\mathrm{P}=0.00386)$ fueron las variables del EOG que se asociaron significativamente con el porcentaje de infección de PGI en las aves analizadas, mientras que las variables: quilla $(\mathrm{P}=0.10416)$, patas $(\mathrm{P}=0.25482)$ y plumaje $(\mathrm{P}=0.30791)$ no resultaron estadísticamente significativas.

Parámetros físicos y bacteriológicos del agua: En los cuatro sitios de estudio, se encontraron, tanto para coliformes fecales como para E. coli, más de 1600 NMP (Numero más Probable) de bacterias por cada $100 \mathrm{ml}$ de agua. De acuerdo con la clasificación de la NSF, la calidad del agua del río Pirro en la época seca, fue mala (entre 25 y 50 ) en los cuatro sitios de estudio; mientras que en la época lluviosa tres de los sitos: Universidad Nacional, Santiago y San Rafael, también lo fueron, sin embargo, la calidad del agua en Guayabal fue muy mala.

Dentro de las variables físicoquímicas medidas en el agua se observó una fluctuación importante en el oxígeno disuelto (0.6 y 4.59 $\mathrm{mg} / \mathrm{l})$, así como variaciones en el índice de calidad del agua entre las épocas climatológicas (Fig. 2).

\section{DISCUSIÓN}

En Costa Rica, este es el primer estudio que describe el EOG y la presencia de PGI en aves silvestres en un ecosistema ribereño

CUADRO 2

EOG, porcentaje y tipo de infección por PGI, en aves del río Pirro, Heredia, 2011

\begin{tabular}{lcccc}
\multicolumn{1}{c}{ Especie } & EOG $(\%)^{1}$ & PI $\left(\mathrm{n}^{+} / \mathrm{n}^{\mathrm{t}}\right)^{2}$ & Infección Simple & Infección Mixta \\
Columbina inca & 0.4 & $50(1 / 2)$ & Co & - \\
Momotus lessonii & 1.1 & $14.2(1 / 7)$ & Co & - \\
Melanerpes hoffmannii & 1.1 & $50(1 / 2)$ & Co & - \\
Troglodytes aedon & 1.1 & $25(1 / 4)$ & Co & I, E, Ch, Ca, Ce, St \\
Turdus grayi & 1.1 & $27.4(17 / 62)$ & - & - \\
Vireo flavoviridis & 0.9 & $33(1 / 3)$ & Co & - \\
Vireo philadelphicus & 0.9 & $33(1 / 3)$ & Co & - \\
Basileuterus rufifrons & 0.8 & $80(4 / 5)$ & I & - \\
Setophaga petechia & 1.0 & $33(1 / 3)$ & Co & - \\
Parkesia noveboracensis & 0.9 & $50(1 / 2)$ & Co & - \\
Cardellina canadensis & 0.9 & $50(1 / 2)$ & $\mathrm{I}$ & - \\
Cardellina pusilla & 0.9 & $100(1 / 1)$ & $\mathrm{I}$ & - \\
Thraupis episcopus & 1.1 & $33(1 / 3)$ & Co & - \\
Saltator coerulescens & 1.4 & $100(1 / 1)$ & Co & - \\
Melozone cabanisi & 1.1 & $33(2 / 6)$ & Co & - \\
Melozone leucotis & 1.1 & $18(2 / 11)$ & Co & \\
Zonotrichia capensis & 1.0 & $17.6(3 / 17)$ & & - \\
\hline
\end{tabular}

(1) EOG (\%) = Examen Objetivo General (\%), Escala utilizada = 0 (Nulo), 0-1 (Bueno), 1.1-1.4 (Regular), 1.5-2 (Malo). (2) $\mathbf{P I}=$ Porcentaje de infección por PGI (muestras positivas/total de muestras).

$\mathbf{I}=($ Isospora spp.), $\mathbf{E}=$ (Eimeria spp.), Ch (Choanotaenia spp.), Ca (Capillaria spp.), Co (Coccidios sin identificar), Ce (cestodos sin identificar), St (Strongylida). 


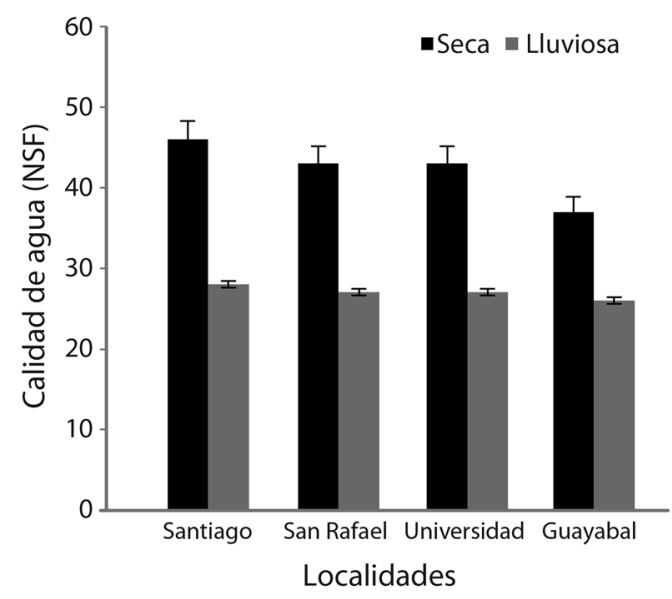

Fig. 2. Índice de Calidad de Agua y D.E (desviación estándar), en los cuatro sitios de estudio durante el año 2011, a lo largo de la microcuenca urbana del río Pirro, Heredia. Rangos del Índice de Calidad de Agua: Excelente (90-100), Buena (70-90), Media (50-70), Mala (25-50), Muy Mala (0-25).

urbano; aunque existe un estudio que valoró la condición corporal de aves silvestres como parámetro de salud en un agroecosistema rural (Hernández et al. 2013).

El EOG determinado para las aves capturadas en el río Pirro, indicó que más del $50 \%$ presentó un buen estado físico de salud. Las aves que presentaron un estado regular y malo de EOG, mostraron signos clínicos como bajo peso corporal, deshidratación y diarrea; siendo aspectos que podrían estar relacionados con la presencia de agentes infecciosos (parásitos, virus y bacterias) como ha sido reportado en aves de vida libre (Samour, 2016).

Los porcentajes de infección por PGI en las especies de aves analizadas, fluctuaron de 14.2 a $100 \%$ (Apéndice digital 3), con valores similares o menores $(<13 \%)$ a los reportados en otros estudios (Hernández et al. 2013; Carrera, 2016).

Los coccidios fueron los PGI con mayor porcentaje de infección (97.6\%), como se ha detectado en aves de vida libre en Irán, Brasil, Estados Unidos, México y Costa Rica (Jiménez et al. 2002; Martínez-Guerrero, PeredaSolís, Rosales-Alférez, \& Herrera-Casio, 2010;
Hernández et al. 2013; Badparva, Ezatpour, Azami, \& Badparva, 2015; Gomes-DosSantos, Oliveira, Barbosa-DeMoura, \& DeSouza-Correia, 2015; Carrera, 2016; González, 2016); sin embargo, los porcentajes de infección varían entre estudios, debido a factores tales como: el diseño del muestreo, especies de aves, dieta y tipo hábitat, entre otros (Zinke, Schnebel, Dierschke, \& Ryll, 2004; Hernández et al. 2013; Badparva et al. 2015; Leung \& Koprivnikar, 2016).

Es importante mencionar, que a pesar de que las muestras de heces no fueron sometidas a esporulación con Dicromato de potasio al 2 \% (Sloss, Kemp, \& Zajac, 1994; Foreyt, 2001), algunos coccidios se detectaron esporulados cuando las heces fueron procesadas por la técnica Sheather. Los coccidios identificados en este estudio fueron Isospora spp. e Eimeria spp. (Apéndice digital 3). El primero estuvo presente en cinco especies de aves, mientras que el segundo se presentó solo en una especie de ave (T. grayi); teniendo el primero un rango amplio de hospedadores respecto al segundo (Samour, 2016). En Costa Rica se ha reportado Isospora spp. e I. zorzai en aves de las familias Icteridae y Turdidae, respectivamente (Picado \& Castillo, 2007; Keller et al. 2012).

Los coccidios tienen un ciclo de vida directo y la resistencia que adquieren en el ambiente favorece su presencia como una posible una fuente de infección para las aves a través del alimento o agua contaminada (Friend \& Franson, 1999; Atkinson, Thomas, \& Hunter; 2008). En la literatura hay un gran número de especies de coccidios en aves de vida libre que han sido reportadas como patógenas, pero usualmente no son de importancia clínica; contrario a lo que sucede en aves de cautiverio, donde con frecuencia se presentan síntomas como: pérdida de peso, diarrea, plumaje erizado y problemas en ciclos reproductivos (Friend \& Franson, 1999; Burbano et al. 2003; Gill \& Paperna, 2008; Samour, 2016).

Por otra parte, los nematodos del grupo Strongylida y Capillaria spp. se detectaron en porcentajes bajos de infección $(2.4 \%)$ y en una única especie de ave de la familia Turdidae; 
contrario a otros estudios, donde los porcentajes de infección fueron mayores $(6 \%$ y $56.1 \%$ ) al valor encontrado (Schoener, 2010; González, 2016). Capillaria spp. es un parásito frecuentemente encontrado en aves, la mayoría de las especies tienen un ciclo de vida directo y parasitan principalmente aves de aviarios y de compañía, como canarios, palomas, gallináceas y guacamayos (Friend \& Franson, 1999). En Costa Rica ha sido identificada C. obsigenata en palomas silvestres (Rodríguez-Ortíz et al. 2004).

El cestodo Choanotaenia spp. (2.4\%) se ha identificado en aves paseriformes (gorriones y yigüirros) de Nigeria y Estados Unidos (Edosomwan \& Ogbonnia, 2014; Carrera, 2016), similar a lo encontrado en este estudio. En Costa Rica $C$. infundibulum ha sido reportado en gallos domésticos (Rodríguez-Ortíz et al. 2004). Este parásito tiene un ciclo de vida indirecto con hospedadores intermediarios como: moscas, escarabajos, termitas y saltamontes (Edosomwan \& Ogbonnia, 2014; Carrera, 2016), que son presas usuales de los yigüirros (Stiles \& Skutch, 2003). La utilización de fuentes de alimentación con artrópodos e insectos podrían representar una importante fuente de contaminación con Choanotaenia spp., para las aves infectadas con este parásito.

A pesar de que se detectaron cestodos sin identificar, estos podrían estar representados por Raillietina, Tetrameres, Hymenolepis Aploparapsis y Anomotaenia, como se ha identificado en aves Paseriformes, Apodiformes y Psittaciformes (Rodríguez-Ortíz et al. 2004; Edosomwan \& Ogbonnia, 2014; Gomes-DosSantos et al. 2015; Carrera, 2016).

En este estudio las infecciones simples predominaron sobre las múltiples, como ha sido reportado en aves Paseriformes, Pisciformes, Psittaciformes y Stringiformes de Costa Rica (Sibaja, 2006; González, 2016), lo cual indica que las aves han estado expuestas a fuentes de infección limitada, favoreciéndose las infecciones con parásitos de ciclos directos (Sibaja, 2006). Sin embargo, cabe mencionar que las infecciones mixtas con PGI en $T$. grayi se presentaron con parásitos de ciclos de vida directos e indirectos, posiblemente por los hábitos alimenticios generalistas de esta especie (Stiles \& Skutch, 2003).

Los PGI en las aves silvestres son bioindicadores de salud del ecosistema, esto debido a la relación que existe entre la presencia de parásitos, el estado físico de las aves y la calidad de su hábitat (Sures, 2001; Costa, Coelho, Bueno, Ferreira, \& Freire, 2010). La cavidad oral y el estado de las alas fueron las variables que presentaron significancia estadística en relación a las infecciones con PGI. Muchas de las aves en su cavidad oral, evidenciaron salivación viscosa, lo que podría indicar un cierto grado de deshidratación, el cual es un signo que algunos nematodos pueden ocasionar en sus hospedadores (Holand, Jensen, Tufto, Saether, \& Ringsby, 2013). En el caso del estado de las alas o plumaje de las aves, se ha demostrado que es uno de los mayores indicadores cualitativos de buena salud (Bitton, Dawson, \& Ochs, 2008; Marichal-Arbona, \& Ávila, 2014). La estructura y composición de la pluma puede verse afectada al existir una mala alimentación y presencia de endoparásitos (Bitton, Dawson, \& Ochs, 2008).

Finalmente, la calidad del agua del río Pirro no fue apta para consumo humano por la contaminación actual y la presencia de coliformes fecales. A pesar de que en este estudio no se encontraron parásitos zoonóticos en las aves analizadas, en un estudio realizado en la microcuenca urbana del río Torres (San José, Costa Rica), se diagnosticaron en las aves, los parásitos zoonóticos Giardia duodenalis y Cryptosporidium parvum. Tanto la microcuenca del Pirro como en la del Torres se presentan problemas con el manejo de las aguas residuales, invasión al cauce del río y pérdida de cobertura (Pérez-Gómez, com. pers). La mala calidad del agua superficial del río, provoca que este recurso disponible para las aves no sea el más apto, ya que utilizan aguas contaminadas con heces humanas (Friend \& Franson, 1999); y a la vez estas aves eventualmente podrían ser transmisores de parásitos con potencial zoonótico.

Los ecosistemas ribereños urbanos que con frecuencia mantienen un alto contenido de 
materia fecal, podrían estar exentos de esto si se da un adecuado manejo de las aguas residuales, lo que permitiría que los ríos urbanos funcionen como corredores biológicos interurbanos aprovechables por aves residentes y migratorias como espacios para refugiarse y alimentarse (Pérez-Gómez, Gastezzi-Arias, \& Vega-Quesada, 2016).

\section{AGRADECIMIENTOS}

Agradecemos a todos los que colaboraron en el trabajo de campo, especialmente a Sergio Quesada, Carola Scholz, Roberto Vargas, Néstor Quesada y Mónica González. Un agradecimiento especial a Federico Herrera por su colaboración en los procesos finales del escrito. Un profundo agradecimiento a Óscar Ramírez por su disposición y apoyo técnico. Al proyecto de Rehabilitación de la microcuenca del río Pirro de la Universidad Nacional de Costa Rica. Finalmente a agradecemos a Zaidett Barrientos y Julián Monge por sus valiosos consejos al manuscrito.

\section{RESUMEN}

En las zonas urbanas, los ríos brindan diversos servicios ecosistémicos, entre ellos, funcionan como corredores biológicos interurbanos, permitiendo a las aves trasladarse y refugiarse en medio de la ciudad. Sin embargo, los altos niveles de contaminación las exponen, e incluso al ser humano, a diversos problemas de salud. De aquí la importancia de realizar diagnósticos ambientales que permitan identificar alteraciones y mitigarlos de forma oportuna. En este estudió se da un primer diagnóstico de la situación parasitaria de las aves silvestres del río Pirro ubicado en un entorno urbano, fragmentado y con altos niveles de contaminación superficial del agua. El muestreo se llevó a cabo en la parte alta y media del río Pirro en Heredia, Costa Rica. Se utilizaron redes de niebla para la captura de las aves, y se les realizó un examen objetivo general (EOG) para revisar su estado de nutrición e hidratación, así como su estado físico; además, se recolectaron muestras de heces para la caracterización de los parásitos gastrointestinales (PGI). Este procedimiento se realizó a 158 aves, correspondientes a 30 especies, se identificaron tres grupos de estos: protozoarios, nematodos y cestodos. Los porcentajes de infección más altos estuvieron representados por los coccidios, con $97.6 \%$, seguido de los nematodos y cestodos con un $2.4 \%$. Dentro del grupo de los coccidios se logró identificar: Eimeria spp., Isospora spp., en los nemátodos: a nivel de grupo Strongylida y a nivel de género: Capillaria spp. Y para los céstodos: Choanotaenia spp. El EOG resultó de regular a bueno en todos los individuos capturados sin embargo, mediante el modelo de regresión lineal general (General Lineal Model) se identificó que la cavidad oral de las aves y el estado del plumaje, podrían ser las variables más relacionadas con el porcentaje de infección por parásitos gastrointestinales, esto debido a que la cavidad oral es la vía principal de ingesta para el ave, así como el estado del plumaje evidencia su vigor.

Palabras clave: aves urbanas; parásitos gastrointestinales; ríos urbanos; aves silvestres.

\section{REFERENCIAS}

Alvarado, V., Bermúdez, T., Romero, M., \& Piedra, L. (2014). Plantas nativas para el control de la erosión en taludes de ríos urbanos. Spanish Journal of Soil Science, 4(1), 99-111.

Arce, E. R. (2005). Uso de protocolos veterinarios en el proceso de reintroducción de la lapa roja (Ara macao) en Costa Rica (Tesis de Licenciatura). Universidad Nacional, Heredia, Costa Rica.

Atkinson, C. T., Thomas, N. J., \& Hunter, D. B. (Eds.). (2008). Parasitic disseases of wild birds. Iowa, United States of America: Wiley-Blackwell.

Azpiri, G., Maldonado, F., \& González, G. (2000). La importancia del estudio de enfermedades en la conservación de la fauna silvestre. Veterinaria México, 31(3), 223-230.

Badparva, E., Ezatpour, B., Azami, M., \& Badparva, M. (2015). First report of birds infection by intestinal parasites in Khorramabad, West Iran. Parasitic Diseases, 39(4), 720-724.

Barrientos, Z. \& Monge-Nájera, J. (2010). Restauración ecológica en la meseta central de Costa Rica. Biocenosis, 23, 20-25.

Bitton, P. P., Dawson, R. D., \& Ochs, C. L. (2008). Plumage characteristics, reproductive investment and assortative mating in tree swallows Tachycineta bicolor. Behavioral Ecology and Sociobiology, 62(10), 1543-1550.

Brejov, G. D. (2014). Semiología Veterinaria Medicina 1. Buenos Aires, Argentina: Facultad de Veterinaria de la Universidad de Buenos Aires (FVET-UBA).

Burbano, P. S., Acosta, D. O., Montaño, J. B., \& Martínez, K. (2003). Parásitos gastrointestinales en las aves de la familia Psittacidae en la Fundación Zoológica de Cali (Cali, Valle del Cauca, Colombia). Medicina Veterinaria, 20(6), 67-72.

Clesceri, L. S., Greenberg, A. E., \& Trussell, R. R. (1989). Standard methods for the examination of water and 
wastewater. Washington, United States of America: American Public Health Association.

Campos, R. F. V. (1997). Estudio coprológico de parásitos en aves de la familia Psittacidae en el centro oficial de rescate de vida silvestre Jardín Gaia (Tesis de Licenciatura). Universidad Nacional, Heredia, Costa Rica.

Carrera, P. D. (2016). Gastrointestinal parasites in captive and free-ranging birds and their possible crosstransmission at the Bristol Zoo Gardens (Tesis de Maestría). University of Bristol, England.

Costa, I. A., Coelho, C. D., Bueno, C., Ferreira, I., \& Freire, E. (2010). Ocorroencia de parasitos gastrintestinais em aves silvestres no municipio de Seropédica, Río de Janeiro, Brasil. Ciencia Animal Brasileira, 11, 914-922.

Edosomwan, E. U., \& Ogbonnia, C. F. (2014). A survey of helminth parasites of wild birds in the university of Benin, Benin-city, Nigeria. African Journals Online, 35(1,2), 462-464.

Foreyt, W. J. (2001). Veterinary Parasitology reference manual. Iowa, United States of America: State University Press.

Freitas, M. F. L., Oliveira, J. B., Cavalcanti, M. D. B., Leite, A. S., Magalhaes, V. S., Oliveira, R. A., \& Evencio-Sobrinho, A. (2002). Parásitos gastrointestinales de aves silvestres en cautiverio en el estado de Pernambuco, Brasil. Parasitología Latinoamericana, 57(1-2), 50-54

Friend, M. \& Franson, C. (1999). Field manual of wildlife diseases: general field procedures and diseases of birds. Washington, United States of America: $U$. S. Geological Survey.

Gill, H. \& Paperna, I. (2008). Proliferative visceral Isospo$r a$ (atoxoplasmosis) with morbid impact on the Israeli sparrow Passer domesticus (biblicus Hartert, 1904). Parasitology Research, 103(3), 493-499.

Gomes-DosSantos, E., Oliveira, J. B., Barbosa-DeMoura, G. J., \& DeSouza-Correia, J. M. (2015). Helmintos intestinales de Amazona amazonica (Psittaciformes: Psittacidae) de vida libre en la región noreste de Brasil. Mexicana de Biodiversidad, 86, 823-825.

González-Acuña, D., Lara, J., \& Cicchino, A. (2009). Nuevos registros de piojos (Insecta: Phthiraptera) en aves domésticas y ornamentales en Chile. Archivos de Medicina Veterinaria, 41(2), 181-184.

González, M. (2016). Presencia de parásitos en aves silvestres (orden Passeriformes) de vida libre de la zona sur de Costa Rica (Tesis de Licenciatura). Universidad Nacional, Heredia, Costa Rica.

Hernández, M., Larramend, M., \& Szczypel, B. (2002). Céstodos más comunes de la gallina doméstica en Cuba. Cubana de Ciencia Avícola, 26, 145-149.
Hernández, S. M., Peters, V. E., Weygandt, P. L., Jiménez, C., Villegas, P., O’Connor, B., Yabsley, M. J., García, M., Riblet, S. M., \& Carroll, C. R. (2013). Do shadegrown coffee plantations pose a disease risk for wild birds? Ecohealth, 10(2), 145-158. Doi: 10.1007/ s10393-013-0837-3

Hinojosa-Sáez, A. \& González-Acuña, D. (2005). Estado actual del conocimiento de helmintos en aves silvestres de Chile. Gayana, 69(2), 241-253.

Holand, H., Jensen, H., Tufto, J., Saether, B. E., \& Ringsby, T. H. (2013). Temporal and spatial variation in prevalence of the parasite Syngamus trachea in a metapopulation of house sparrows (Passer domesticus). Parasitology, 140(10), 1275-1286.

Jiménez, A., Wearing, J., Hernández, J., Di Mare, M., Villareal, J., Cedeño, Y., \& Ramírez, O. (2002). Parásitos en palomas silvestres: Collareja \& Alablanca (I parte). Boletín de Parasitología (Costa Rica), 3(2), 1.

Keller, S. P., Yabsley, M. J., Gibbs, S. E. J., McGraw, S. N., \& Hernández, S. M. (2012). A new Isospora species of passerines in the family Turdidae from Costa Rica. Parasitology, 98(1), 167-169. Doi: 10.1645/ GE-2721.1

Leung, T. L. F. \& Koprivnikar, J. (2016). Nematode parasite diversity in birds: the role of host ecology, life history and migration. Animal Ecology, 85(6), 14711480. Doi:10.1111/1365-2656.12581

List, M. C. S. \& Solano, O. E. G. (2001). Práctica dirigida en el manejo veterinario de primates no humanos en cautiverio (Tesis de Licenciatura). Universidad Nacional, Heredia, Costa Rica.

Loot, G., Park, Y. S., Lek, S., \& Brosse, S. (2006). Encounter rate between local populations shapes host selection in complex parasite life cycle. Biological Journal of the Linnean Society, 89(1), 99-106.

Marín-Gómez, S. Y. \& Benavides-Montaño, J. A. (2007). Parásitos en aves domésticas (Gallus domesticus) en el noroccidente de Colombia. Veterinaria y Zootecnia, 1(2), 43-51.

Marichal-Arbona, E. \& Denis-Ávila, D. (2014). Variaciones sexuales y eterias de la coloración del plumaje de Erythrura gouldiae (Passeriformes: Estrildidae) en cautiverio. Cubana de Ciencias Biológicas, 3(1), 68-80.

Martínez-Guerrero, J. H., Pereda-Solís, M. E., RosalesAlférez, F., \& Herrera-Casio, H. (2010). Parásitos gastrointestinales del guajolote silvestre de Gould (Meleagris gallopavo mexicana): abundancia, distribución, prevalencia y diversidad. Agrociencia, 44(5), 541-547.

Monge-Nájera, J. (2013). Potencia de las capitales provinciales de Costa Rica para albergar corredores biológicos urbanos. Ambientico, 232-233, 75-80. 
Oram, B. (2013). Online Calculator for Determing a Water Quality Index. Dallas, United States of America: National Sanitation Foundation (NSF). Recuperado de http:/www.water-research.net/ index.php/water-treatment/water-monitoring/ monitoring-the-quality-of-surfacewaters

Papazahariadau, M., Diakou, A., Papadopoulos, E., Georgopoulou, I., Komnenou, A., \& Antoniadou-Sotiriadou, K. (2008). Parasites of the digestive tract in free-ranging birds in Greece. Natural History, 42(5-8), 381-398.

Pérez-Gómez, G., Gastezzi-Arias, P., \& Vega-Quesada, A. (2016). Avifauna poco frecuente en la microcuenca del río Torres, San José, Costa Rica. Zeledonia, 20(2), 20-27.

Picado, P. \& Castillo, M. (2007). Determinación de Salmonella spp. y endoparásitos en zanates (Quiscalus mexicanus) del parque de Cañas, Guanacaste. Costarricense de Salud Pública, 16(31), 1400-1429.

Posada, S. L \& Rosero, R. (2007). Comparación de modelos matemáticos: una aplicación en la evaluación de alimentos para animales. Colombiana de Ciencias Pecuarias, 20, 141-148.

R Development Core Team. (2008). R: A language and environment for statistical computing. Vienna, Austria: R Foundation for Statistical Computing.

Ralph, C. J., Geupel, R., Pyle, P., Martin, T. E., DeSante, D. F., \& Milá, B. (1996). Manual de métodos de campo para el monitoreo de aves terrestres. California, United States of America: Department of Agriculture.

Rodríguez-Ortíz, B., García-Prieto, L., \& Pérez-Ponce de León, G. (2004). Checklist of the helminth parasites of vertebrates in Costa Rica. Biología Tropical, 52(2), 313-354.

Rojas, P. Y., Larramendy, R., Sánchez, I. Y., Mena, R. R., \& Neyra, R. D. (2009). Presencia de Phylophthalmus sp
(Trematoda: Philophthalmidae) en gallinas criadas en traspatio. Salud Animal, 31(3), 180-182.

Romero, M., Piedra, L., Villalobos, R., Marín, R., \& Núñez, F. (2011). Evaluación ecológica rápida de un ecosistema urbano: El caso de la microcuenca del río Pirro, Heredia, Costa Rica. Geográfica de América Central, 47, 41-70.

Schoener, E. R. (2010). Gastrointestinal parasites in endemic, native, and introduced New Zealand passerines with a special focus on Coccidia (Tesis de Maestría). Massey University, Palmerston, New Zealand.

Sibaja, K. D. (2006). Identificación de los parásitos gastrointestinales y ectoparásitos de animales silvestres en cautiverio en Costa Rica (Tesis de Licenciatura). Universidad Nacional, Heredia, Costa Rica.

Sloss, M. W., Kemp, R. L., \& Zajac, A. M. (1994). Veterinary Clinical Parasitology. Iowa, United States of America: State College Press.

Stiles, G. \& Skutch, A. F. (2003). Guía de Aves de Costa Rica. Heredia, Costa Rica: Instituto Nacional de Biodiversidad.

Sures, B. (2001). The use of fish as bioindicators of heavy metals in aquatic ecosystems: a review. Aquatic Ecology, 35, 245-255.

Tomás-Cabedo, G., Merino, S., Moreno, J., Morales, J., \& Martínez-De La Puente, J. (2007). Impact of blood parasites on immunoglobulin level and parental effort: a medication field experiment on a wild passerine. Functional Ecology, 21(1), 125-133.

Zinke, A., Schnebel, B., Dierschke, V., \& Ryll, M. (2004). Prevalence and intensity of excretion of coccidial oocysts in migrating passerines on Helgoland. Ornithology, 145(1), 74-78. 